Check for updates

Cite this: RSC Adv., 2018, 8, 24434

\title{
Hesperetin inhibits Eca-109 cell proliferation and invasion by suppressing the PI3K/AKT signaling pathway and synergistically enhances the anti- tumor effect of 5-fluorouracil on esophageal cancer in vitro and in vivo
}

\begin{abstract}
Dandan Wu, (D) Jiao Li, (D) Xue Hu, (D) Jingjing Ma (D) and Weiguo Dong (D)*
Previously, we reported that hesperetin exhibited pro-apoptotic activity against esophageal cancer in vitro and in vivo. Here, we examined whether hesperetin inhibits cell proliferation and invasion and synergistically enhances the anti-tumor effect of 5-fluorouracil (5-FU) in esophageal cancer. Flow cytometry, EdU staining, and transwell invasion assays using Eca-109 cells showed that hesperetin induced cell cycle arrest at the G0/G1 phase and inhibited cell proliferation and invasion significantly. Moreover, hesperetin suppressed the expression of phosphorylated PI3K/AKT, cyclin D1, MMP-2, and MMP-9 and increased phosphorylated PTEN and p21 in Eca-109 cells. Hesperetin combined with 5-FU inhibited cell growth more effectively than did either drug alone in Eca-109 cells and in a xenograft mouse model. Hoechst 33258, Annexin V-PE/7-ADD double staining and TUNEL assay showed more apoptotic cells in the combination treatment group than in either individual treatment group. The combination downregulated protein levels of $\mathrm{Bcl}-2$ and up-regulated those of $\mathrm{Bax}$, cleaved caspase-3, and cleaved caspase-9 more effectively than did either drug alone. These data suggest that hesperetin inhibited esophageal cancer cell proliferation and invasion by suppressing the PI3K/AKT signaling pathway. In conclusion, 5-FU and hesperetin exerted synergistic antitumor effects in vivo and in vitro and could constitute a novel therapeutic approach for esophageal cancer.
\end{abstract}

Received 31st January 2018

Accepted 25th April 2018

DOI: $10.1039 / c 8 r a 00956 b$

rsc.li/rsc-advances studies have demonstrated that hesperetin can be an effective antitumor agent by inhibition of cell growth and invasion and induction of cell apoptosis in various cancers. ${ }^{6}$ Choi reported that hesperetin induced G1-phase cell cycle arrest in MCF-7 human breast cancer cells by regulating CDK4 and p21 expression. ${ }^{7}$ Alshatwi et al. showed that the anticancer effect of hesperetin on human cervical cancer cells is mediated through the cell cycle arrest, death receptor, and mitochondrial pathways. ${ }^{8}$ We also have demonstrated that hesperetin can induce apoptosis of esophageal cancer cells via a mitochondrial pathway mediated by increasing intracellular reactive oxygen species (ROS). ${ }^{9}$ ROS can also act as a signal transducer to modulate many other signaling pathways, such as the ERK, JNK, MAPK, and Akt pathways. ${ }^{10-14}$

In particular, the PI3K/Akt pathway is frequently activated in diverse cancers, including esophageal cancer, and is involved in regulating many physiologic processes, such as cell survival, differentiation, and apoptosis. ${ }^{15,16}$ PI3K activation could catalyze the synthesis of lipid second messenger phosphatidylinositol-3,4,5-trisphosphate (PIP3), which subsequently recruits and activates Akt. An overactive PI3K/Akt pathway could reduce the process of apoptosis by phosphorylating apoptotic signaling proteins directly and promote tumor cell cycle progression by modulating the activity of transcription 
factors. Phosphatase and tensin homolog (PTEN), a tumor suppressor, can negatively regulate the synthesis of PIP3 and thereby inhibits the activation of the Akt survival signaling pathway. ${ }^{17}$ Suppressing the PI3K/Akt signaling pathway may be an effective treatment for esophageal cancer. Some studies have shown that hesperetin can modulate the PI3K/Akt signaling pathway. ${ }^{\mathbf{1 8 - 2 0}}$ We were therefore interested in studying the antiproliferation and anti-invasion efficacy of hesperetin in esophageal cancer cells via inhibition of the PI3K/Akt pathway.

5-Fluorouracil (5-FU), belonging to the class of antimetabolism, cycle specific drugs, mainly inhibits the synthesis of DNA and RNA. 5-FU plays a role in several pathways, but principally as a thymidylate synthase (TS) inhibitor which is responsible for the synthesis of the pyrimidine thymidine. Pyrimidine thymidine is a nucleoside required for DNA replication. ${ }^{21}$ For the past several decades, 5-FU has been a first-line treatment for esophageal cancer. $^{3}$ However, single-agent chemotherapy is not ideal for the treatment of esophageal cancer because of the frequent occurrence of cell toxicity, drug resistance, and other adverse effects. 5-FUbased drug combination therapy has been employed in efforts to reduce toxicity and resistance. ${ }^{22}$ Although combination therapy has yielded better adverse effect profiles, drug resistance and cell toxicity remain major issues. New combination regimens with greater therapeutic effects and lower toxicity are urgently needed. In the previous study, ${ }^{9}$ we found that hesperetin induced esophageal cancer cell apoptosis by promoting ROS production, regulating the expression of Bcl-2 protein family, and then activating mitochondria-mediated apoptosis pathway. Hesperetin might make tumor cells more sensitive to other chemotherapeutic drugs or stimuli. As 5-FU and hesperetin act on different targets of esophageal cancer cells, we predicted that the combined use of these two drugs can improve the curative effect. In this study, we also evaluated the synergistic effect of hesperetin and 5-FU against esophageal cancer in vitro and in vivo.

\section{Materials and methods}

\section{Cell culture}

The human esophageal squamous cell carcinoma cell line Eca109 was obtained from the China Center for Type Culture Collection (CCTCC). The cells were cultured in Dulbecco modified Eagle medium/F-12 medium (HyClone) supplemented with $10 \%$ fetal bovine serum (Gibco) plus $1 \%$ antibiotics $\left(100 \mathrm{U} \mathrm{ml}^{-1}\right.$ penicillin and $100 \mu \mathrm{g} \mathrm{ml} \mathrm{m}^{-1}$ streptomycin) in a humidified incubator containing $5 \% \mathrm{CO}_{2}$ at $37{ }^{\circ} \mathrm{C}$.

\section{Reagents}

Hesperetin was purchased from Sigma-Aldrich and dissolved in dimethyl sulfoxide (DMSO) as previously described. ${ }^{9}$ PTEN, phosphorylated PTEN, PI3K, Akt, phosphorylated Akt, procaspase-3, cleaved caspase-3, procaspase-9, cleaved caspase9, Bax, Bcl-2, cyclin D1, MMP-2, MMP-9, and glyceraldehyde-3phosphate dehydrogenase (GAPDH) rabbit monoclonal antibodies were purchased from Cell signaling Technology. Horseradish peroxidase-conjugated anti-rabbit immunoglobulin $\mathrm{G}$ was purchased from LI-COR Biosciences.

\section{EdU assay}

Cell proliferation was measured by using the Cell-Light EdU Apollo 488 In Vitro Imaging kit (RiboBio). EdU is a thymidine analogue which can substitute for thymidine to penetrate into the DNA molecule during DNA replication period. Briefly, Eca109 cells were treated with various concentrations of hesperetin $(0,100,200,300 \mu \mathrm{M})$ for $24 \mathrm{~h}$. Then, the cells were digested and seeded into a 96-well plate $\left(2 \times 10^{4}\right.$ cells per well $)$ supported with $10 \mu \mathrm{M}$ EdU-containing medium. After incubation for $24 \mathrm{~h}$, the cells were fixed, incubated with glycine, and EdU-stained following the manufacturer's protocol. Subsequently, the cells were co-stained with Hoechst 33342 for $30 \mathrm{~min}$. The proportion of the cells that incorporated EdU was determined with a fluorescent microscope.

\section{Cell cycle assay}

Cell cycle analysis was detected by flow cytometry. Briefly, Eca109 cells were allowed to adhere in six-well plates. Before treatment with hesperetin, the cells were cultured in serum-free culture medium for $12 \mathrm{~h}$. After $24 \mathrm{~h}$ of incubation, the cells were harvested and fixed with $70 \%$ ice-cold ethanol overnight at $4{ }^{\circ} \mathrm{C}$, followed by propidium iodide (PI) staining. The different cell cycle phases were analyzed using a FACScan flow cytometer (BD FACSCalibur). The data presented are representative of those obtained in at least three independent experiments.

\section{Transwell invasion assay}

After treated with different concentrations of hesperetin $(0,100$, $200,300 \mu \mathrm{M}$ ) for $24 \mathrm{~h}$, the Eca-109 cells were digested and suspended. A total of $100 \mu \mathrm{l}$ of the cell suspension $\left(1 \times 10^{4}\right.$ cells $)$ was seeded into the upper chamber of a Transwell insert (polycarbonate membranes with $8 \mu \mathrm{m}$ pores; Corning) precoated with Matrigel (BD Biosciences). $24 \mathrm{~h}$ later, the cells in the upper chamber were scrubbed out with cotton swabs. Then, the inserts were fixed with $4 \%$ paraformaldehyde for $15 \mathrm{~min}$ and stained with $0.1 \%$ crystal violet for $5 \mathrm{~min}$. The cells passing through the chamber were observed under an inverted microscope, and eight fields of view were randomly selected to count cells.

\section{Cell viability assay}

Cell proliferation and viability were assessed using CCK-8 assay (Dojindo) as previously described. Briefly, Eca-109 cells $\left(2 \times 10^{4}\right.$ cells per well) were seeded into a 96-well plate. The cells were allowed to adhere for $24 \mathrm{~h}$ and then incubated with $0,25,50$, $100,200,400$, or $800 \mu \mathrm{M}$ hesperetin; $0,2.5,5,10,20,40$, or 80 $\mu \mathrm{M} 5$-FU; or the combination of hesperetin and 5-FU for 24 and $48 \mathrm{~h}$, separately. Afterwards, $10 \mu \mathrm{l}$ of CCK- 8 dye was added to each well. A microplate reader (PerkinElmer) was used to measure the absorbance of each sample at $450 \mathrm{~nm}$.

\section{Apoptosis detection}

Firstly, cell apoptosis was detected by employing Hoechst 33258 dye (Beyotime) as previously described. The cells were treated with hesperetin, 5-FU, or both for $48 \mathrm{~h}$. Subsequently, cells were fixed and stained. The images were observed under an inverted 
fluorescence microscope (Olympus). Eight random fields of view were selected to count cells. Then, Annexin V-PE/7-ADD double staining with flow cytometry was used to further detect cell apoptosis. After Eca-109 cells were exposed to different drugs for $24 \mathrm{~h}$, they were co-stained with $5 \mu \mathrm{l}$ Annexin V-PE and $10 \mu \mathrm{l}$ 7-ADD prior to flow cytometric analysis. The density plots illustrate four cell populations (live, early apoptotic, late apoptotic and dead) according to their fluorescence characteristics. Live cells are both PE and 7-ADD negative, early apoptotic cells are PE positive and 7ADD negative, late apoptotic are both positive, and dead cells are $\mathrm{PE}$ negative and 7-ADD positive.

\section{Western blot analysis}

Eca-109 cells were collected after the indicated treatments, and total cell proteins were extracted in radioimmunoprecipitation assay (RIPA) buffer supplemented with protease inhibitor cocktails (PIC) for $30 \mathrm{~min}$ on ice. Protein concentrations were measured using a BCA protein assay kit (Beyotime) according to the manufacturer's instructions. The proteins were resolved by sodium dodecyl sulfate loading buffer and denatured at $95{ }^{\circ} \mathrm{C}$ for $5 \mathrm{~min}$. A total of $30 \mu \mathrm{g}$ proteins were separated on a $10 \%$ sodium dodecyl sulfate polyacrylamide gel using electrophoresis and then transferred onto Immobilon-PVDF membranes (Millipore) using a wet transfer system. Membranes were blocked for $1 \mathrm{~h}$ with $5 \%$ non-fat
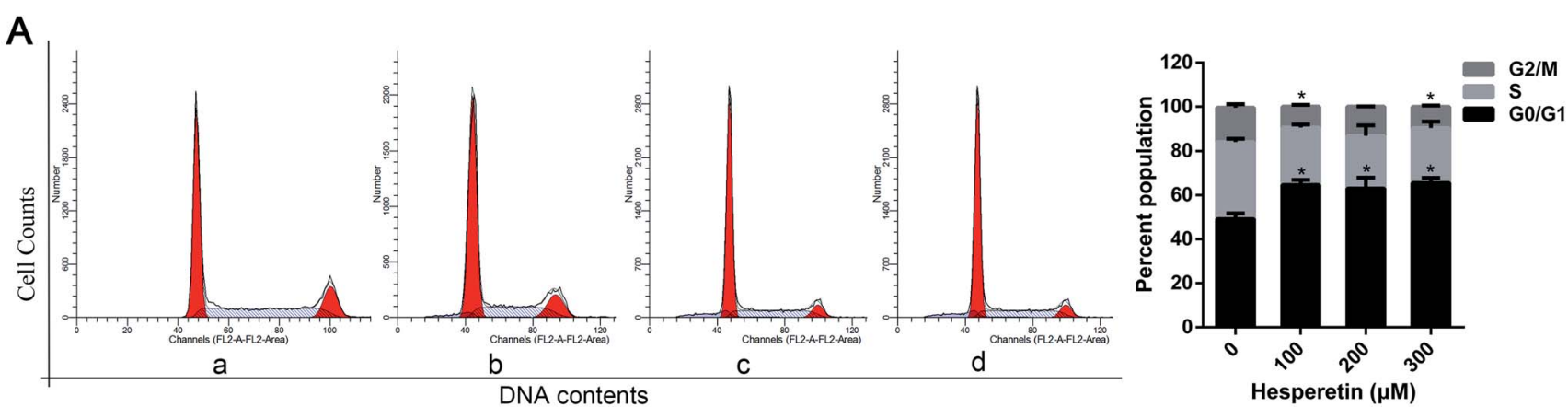

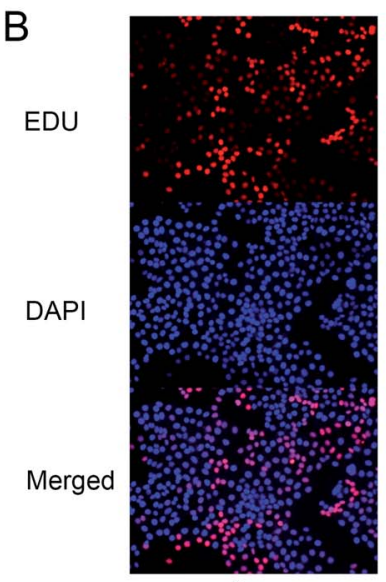

a

C

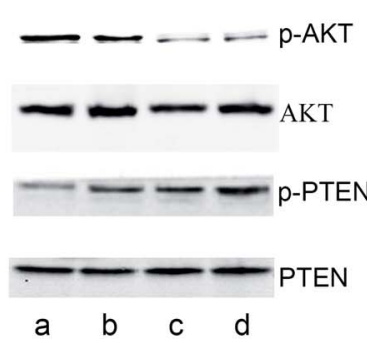

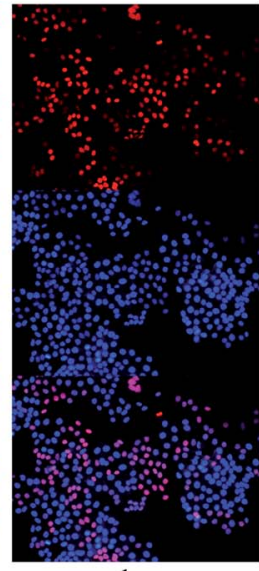

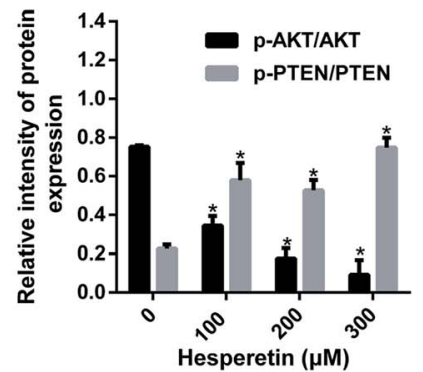

C
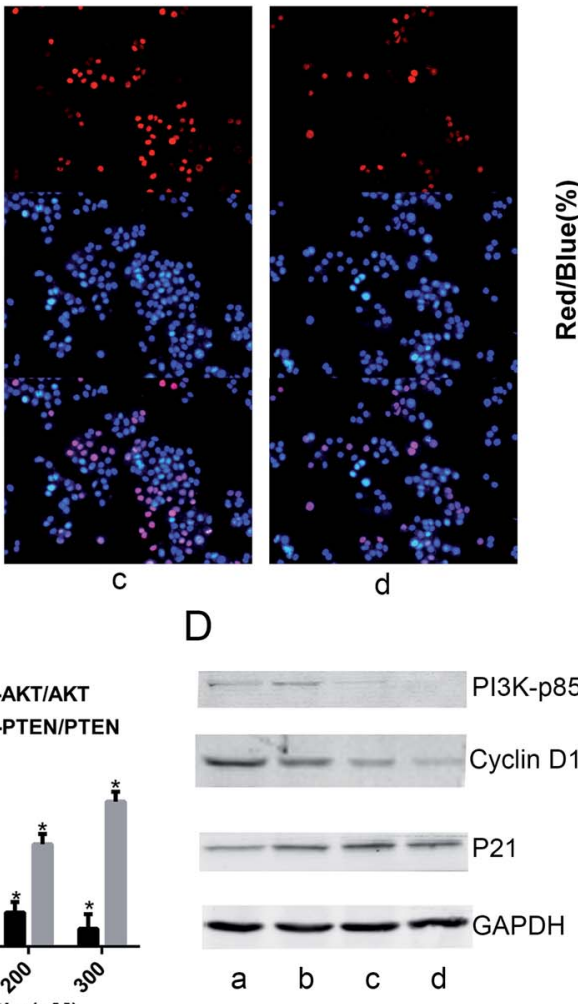
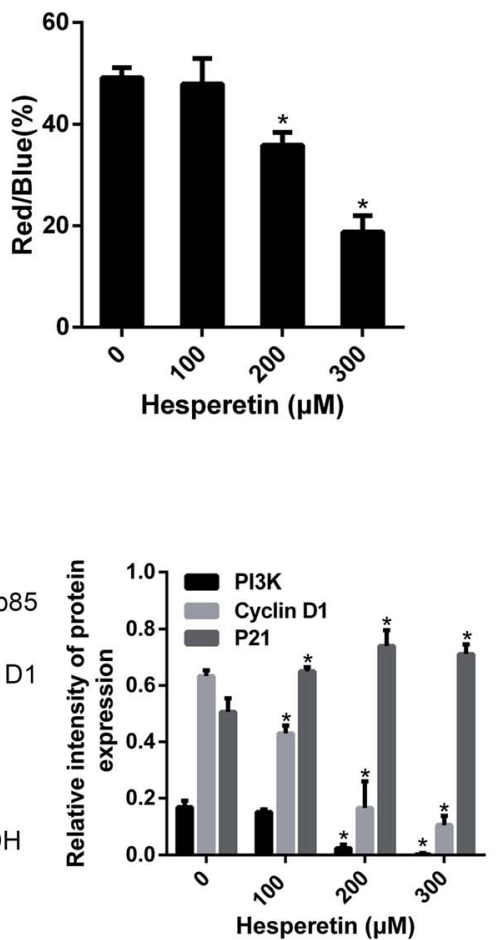

Fig. 1 Hesperetin inhibited Eca-109 cell proliferation. (A) Eca-109 cells were treated with vehicle or hesperetin (100, 200, or 300 $\mu \mathrm{M})$ for $24 \mathrm{~h}$ and then assessed for cell cycle distribution ( $a$, control; b, hesperetin at $100 \mu \mathrm{M} ; \mathrm{c}$, hesperetin at $200 \mu \mathrm{M}$; $d$, hesperetin at $300 \mu \mathrm{M}$. $a, b, c$ and d is same in $C$ and D). Representative individual cell cycle distributions are shown. (B) EdU immunofluorescence staining confirmed the effect of hesperetin on cell proliferation (original magnification, $100 \times$ ). The red/blue ratio was analyzed. Eight random fields were counted for every group. (C) $p$-Akt, Akt, $p$-PTEN, and PTEN expression levels were detected by western blot in untreated and treated cells. The ratios of $p$-Akt/Akt and p-PTEN/PTEN were calculated. (D) The expression levels of PI3K-p85, cyclin D1, and P21 were detected by western blot. Quantitative analysis of each band intensity was normalized to GAPDH and expressed as means \pm standard error of the mean (SEM) $(n=3)$. * $p<0.05$ versus control. 
dry milk and then incubated with various primary antibodies (working concentration, $1: 1000$ ) overnight at $4{ }^{\circ} \mathrm{C}$. After washed with TBST, the PVDF membranes were incubated with secondary antibody (horseradish peroxidase-conjugated anti-rabbit immunoglobulin G, $1: 10$ 000) for $1 \mathrm{~h}$ at room temperature. Immunoreactive protein bands were visualized with an Odyssey Infrared Imaging System (LI-COR Biosciences).

\section{Xenograft tumor model}

Female BALB/c nude mice (5-6 weeks old) purchased from Hunan SJA Laboratory Animal Company were maintained under specificpathogen-free conditions. Before tumor implantation, the mice were allowed to get acclimatized for 1 week. This study was performed in strict accordance with the NIH guidelines for the care and use of laboratory animals (NIH Publication no. 85-23 Rev. 1985) and was approved by the Animal Care and Use Committees of Renmin Hospital of Wuhan University (Wuhan, China). Briefly, $5 \times 10^{6}$ cells suspended in $200 \mu$ l of phosphate-buffered saline were inoculated subcutaneously into the upper right flank of the nude mice. When the tumor diameter reached approximately 5-6 $\mathrm{mm}$, the mice were pooled and divided into four groups (six mice per group), then they received intraperitoneal injection of hesperetin $\left(60 \mathrm{mg} \mathrm{kg}^{-1}\right), 5-\mathrm{FU}\left(10 \mathrm{mg} \mathrm{kg}^{-1}\right)$, hesperetin $\left(60 \mathrm{mg} \mathrm{kg}^{-1}\right)$ plus 5-FU (10 mg kg ${ }^{-1}$ ), or saline every 3 days. Mouse weight and tumor volume were measured every 3 days. Tumor volume in $\mathrm{mm}^{3}$ was determined by measuring the longest diameter $(D)$ and shortest diameter $(d)$ and calculated as $0.5 \times d^{2} \times D$. All the mice were euthanized after 3 weeks of treatment. Blood was collected to detect the levels of serum alanine aminotransferase, aspartate aminotransferase, blood urea nitrogen, and serum creatinine. The tumor xenografts were extracted for hematoxylin and eosin (HE) staining and terminal deoxynucleotidyl transferase dUTP nick-end labeling (TUNEL) assay as described before. ${ }^{9}$ Other important organs, including livers, lungs, kidneys, and brains, were also harvested for HE staining to identify any metastases.

\section{Statistical analysis}

Data were analyzed using an unpaired two-tailed Student $t$-test. A $p$ value less than 0.05 was considered to indicate statistical significance.

\section{Results}

\section{Hesperetin inhibits Eca-109 cell proliferation}

A previous study suggested that hesperetin inhibits Eca-109 cell growth in a concentration- and time-dependent manner. Here,

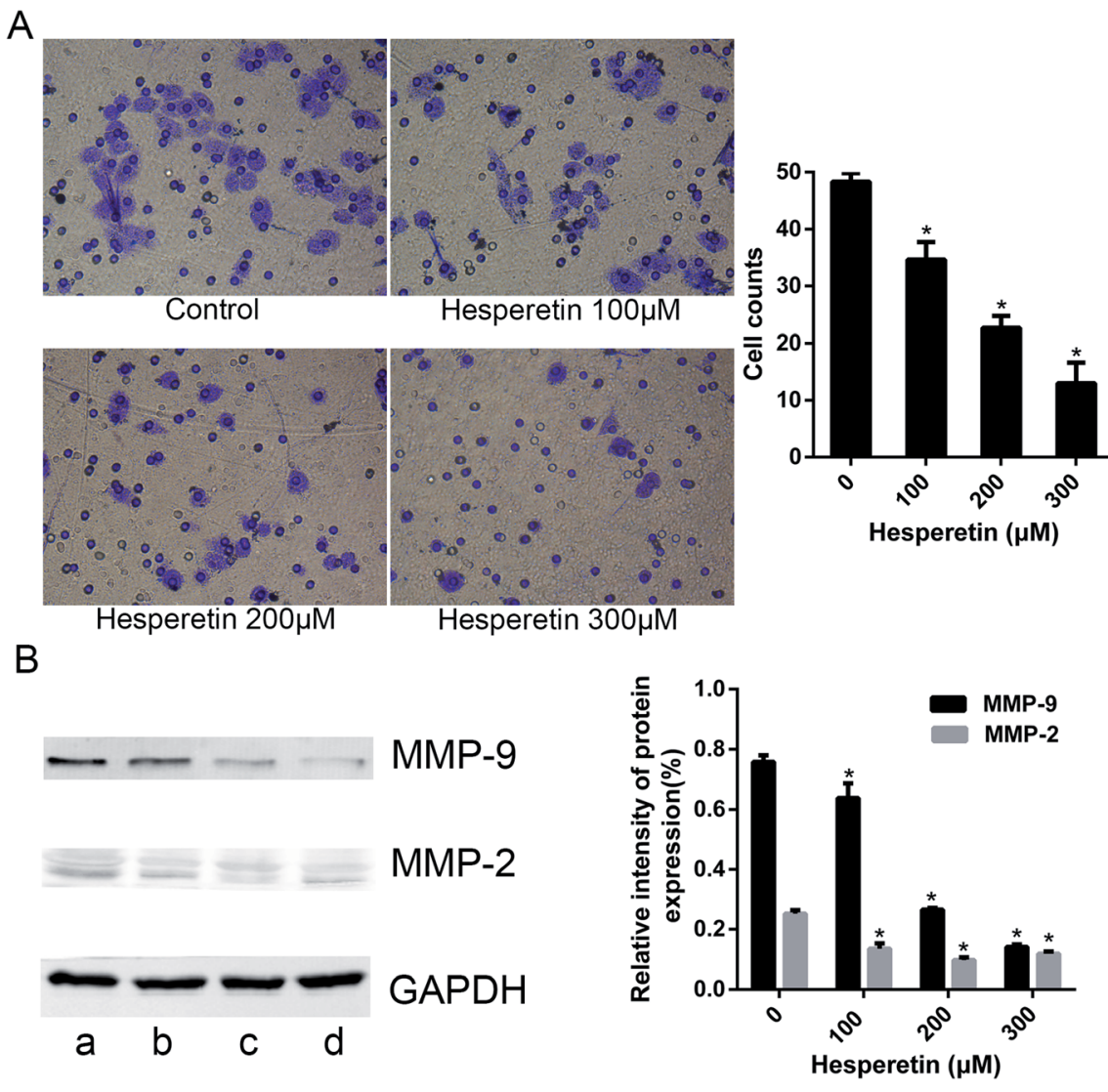

Fig. 2 Hesperetin inhibited Eca-109 cell invasion. (A) Transwell assay was conducted to assess the effect of hesperetin on Eca-109 cells invasion capacity. The number of cells that invaded through the membrane pre-coated with Matrigel was counted under a light microscope with $100 \times$ magnification. (B) The expression levels of invasion-associated proteins (MMP-2, MMP-9) were detected by western blot ( $a$, control; $b$, hesperetin at $100 \mu \mathrm{M} ; \mathrm{c}$, hesperetin at $200 \mu \mathrm{M}$; d, hesperetin at $300 \mu \mathrm{M})$. Data are presented as means $\pm \operatorname{SEM}(n=3)$ and analyzed with the Student $t$-test. * $p$ $<.05$ versus control. 
we found that high-dose hesperetin $(200$ or $300 \mu \mathrm{M})$ induced cell cycle arrest at the G0/G1 phase in Eca-109 cells (Fig. 1A). We also examined the effect of hesperetin on cell proliferation using an EdU assay, which detects nucleotide analogue incorporation into replicating DNA in place of thymidine. As shown in Fig. 1B, 100 $\mu \mathrm{M}$ hesperetin did not inhibit EdU incorporation significantly, but the percentage of EdU-positive cells was much lower in cells treated by 200 or $300 \mu \mathrm{M}$ hesperetin than in controls. To investigate the mechanisms underlying altered cell growth, western blot analysis was performed to determine protein expression associated with the PI3K/Akt signaling pathway. Fig. 1C showed that hesperetin notably down-regulated Akt phosphorylation in a dose-dependent manner while enhancing phosphorylation of PTEN. As shown in Fig. 1D, the expression of PI3K-p85 decreased after hesperetin treatment $(p<0.05)$. Cell cycle progression is controlled by cell cycle regulatory proteins that include p21 and cyclin D1, and we found that the expression levels of p21 significantly increased, while cyclin D1 decreased (Fig. 1D).

\section{Hesperetin inhibits Eca-109 cell invasion}

A transwell assay showed that the invasion abilities of Eca-109 cells were decreased significantly in those treated with hesperetin compared with controls $(p<0.05$; Fig. 2A). As the concentration of hesperetin increased, the invaded cells became less. In addition, we evaluated the expression levels of the invasion-associated proteins MMP-2 and MMP-9. As Fig. 2B showed, MMP-2 and MMP-9 decreased dramatically after hesperetin treatment $(p<0.05)$.

\section{Hesperetin and 5-FU synergistically induce Eca-109 cell apoptosis}

To investigate the synergistic effect of hesperetin and 5-FU on Eca-109 cells, the inhibitory effect of treatment on cell proliferation was determined by the CCK-8 assay. The cells were treated with hesperetin and 5-FU individually or in combination. As shown in Fig. 3A and C, hesperetin combined with 5-FU (molar ratio $10: 1$ ) inhibited Eca-109 cell proliferation more effectively than either compound alone did $(p<0.05)$. The Calcusyn 2.0 software analyzed the combination index (CI). Fig. 3B and D showed that CI was less than 1 in most concentration ranges, which meant hesperetin and 5-FU exerted synergistic anti-proliferative effect on Eca-109 cells.

Cell apoptosis in each group was evaluated by Hoechst 33258 staining. The apoptotic cells were identified by nuclear morphology changes such as bright-blue fluorescent, condensed nuclei or fragmented nuclei. As shown in Fig. 4A,
A

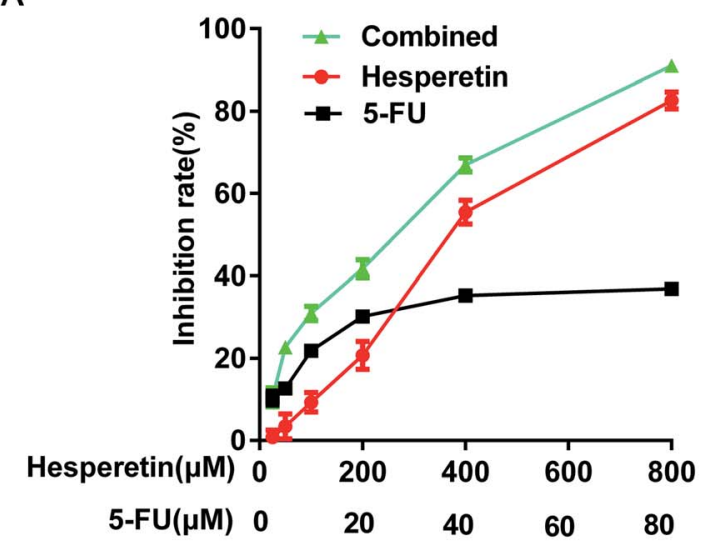

C

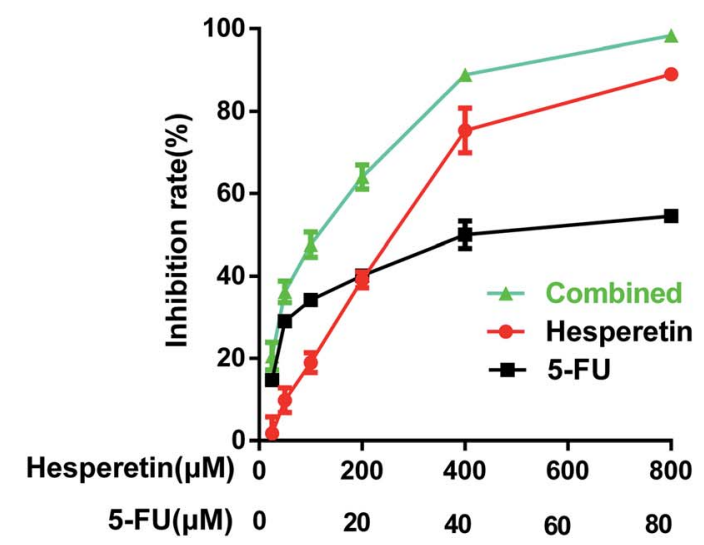

B

$\mathrm{Fa}-\mathrm{Cl}$ plot

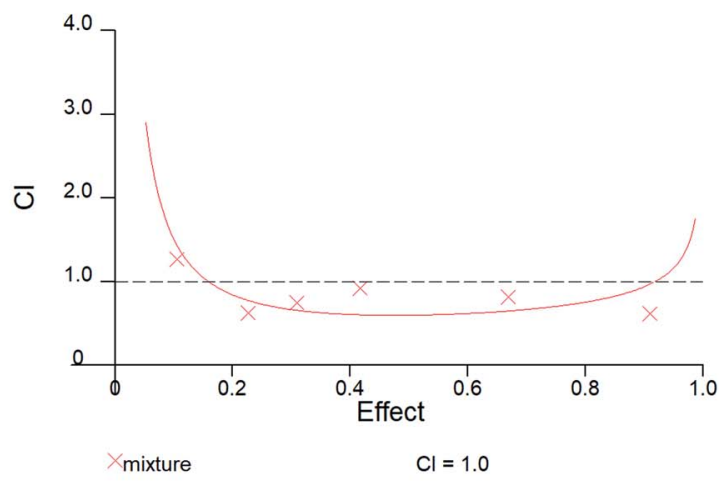

Fa-Cl plot

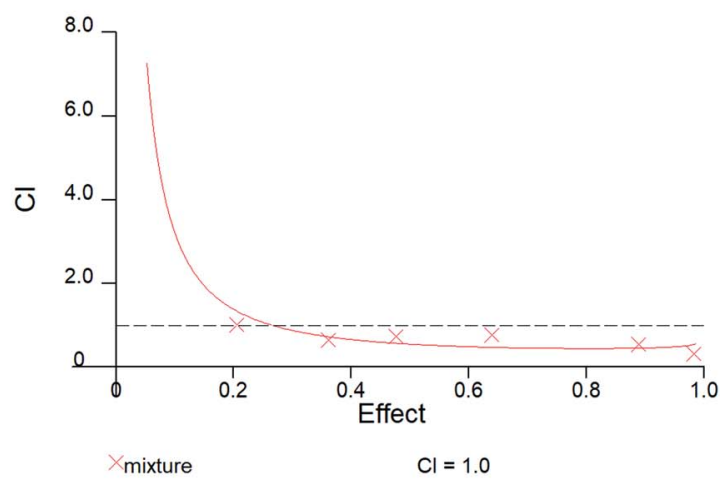

Fig. 3 The effects of hesperetin and 5-FU on Eca-109 cell viability. Representative results for inhibition rate were evaluated after $24 \mathrm{~h}(\mathrm{~A})$ and $48 \mathrm{~h}$ (C) treatment. (B) $\mathrm{Cl}$ at $24 \mathrm{~h}$. (D) $\mathrm{Cl}$ at $48 \mathrm{~h}$. The $\mathrm{Cl}$ values were determined using the Calcusyn 2.0 software. $\mathrm{Cl}=1$, cumulative effect; $\mathrm{Cl}<1$, synergistic effect; and $\mathrm{Cl}>1$, antagonistic effect. 
A

a

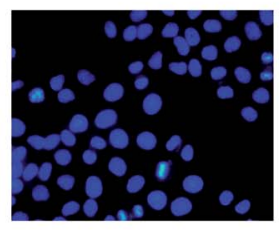

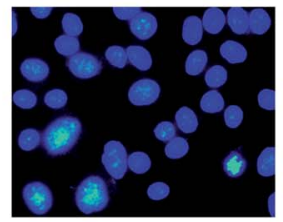

C

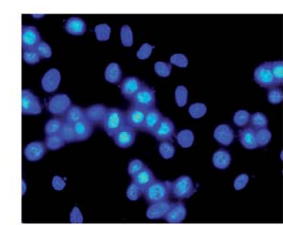

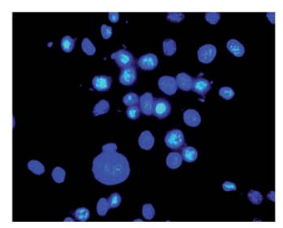

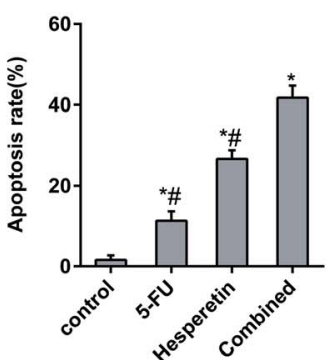

d

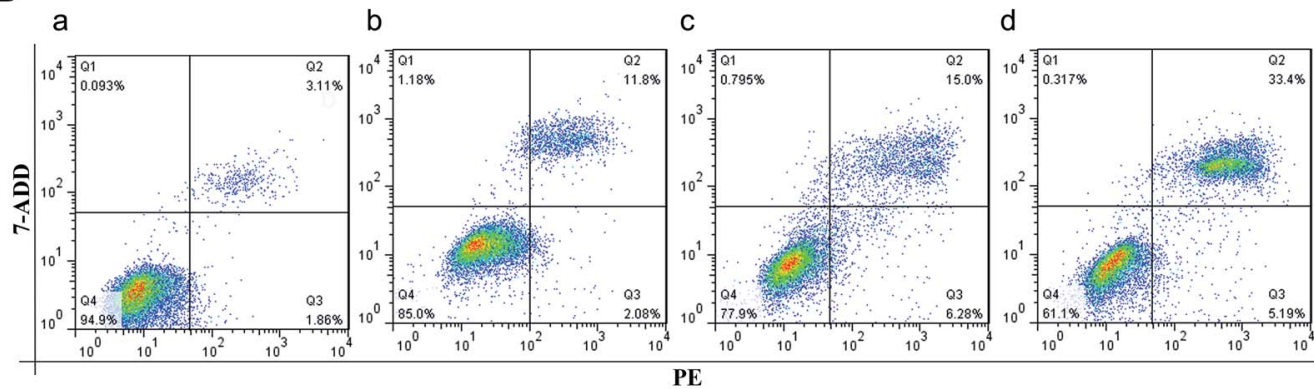

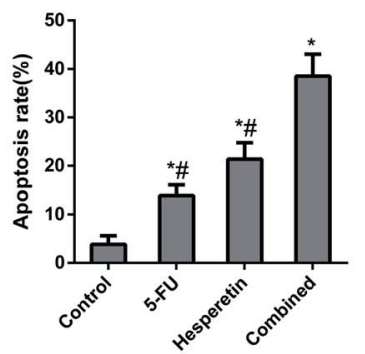

C

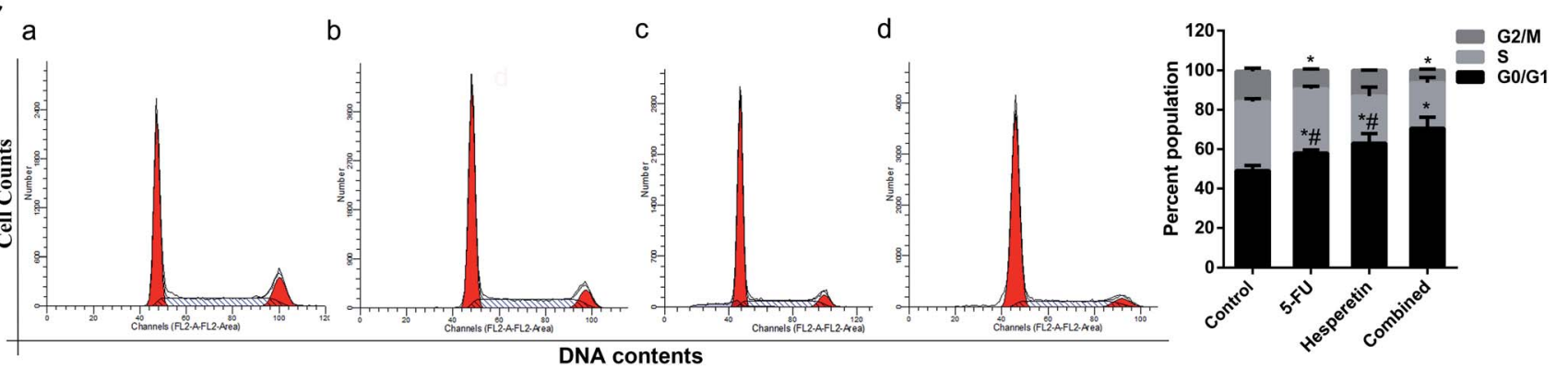

Fig. 4 Hesperetin and 5-FU synergistically induced apoptosis of Eca-109 cells. (A) Apoptosis-related morphologic changes in the cells were detected using Hoechst 33258 staining (200x) (a, control; b, 5-FU at $20 \mu \mathrm{M}$; c, hesperetin at $200 \mu \mathrm{M}$; d, $20 \mu \mathrm{M} 5$-FU + 200 $\mu$ M hesperetin. a, b, c and $d$ is same in $B$ and $C$ ). The apoptosis rate was defined as the ratio of the number of apoptotic cells to the total number of cells. Eight random fields were counted for every dish. (B) Cell apoptosis was analyzed by flow cytometry with Annexin V-PE/7-ADD double staining. The apoptosis rate is expressed as mean $\pm S D$. (C) Cell cycle was analyzed after treatment with hesperetin and 5-FU individually or combined for $24 \mathrm{~h}$. Cell cycle distribution was calculated. ${ }^{*} p<0.05$ versus control. ${ }^{*} p<0.05$ versus combination.

compared with the control group, more swollen and bright-blue fluorescent nuclei were seen in the group treated with 5-FU only, and there was more condensed chromatin in the group treated with hesperetin only. The percentage of apoptotic cells induced by the combined treatment was greater than that induced by either individual treatment $(p<0.05)$. We further verify the synergistic pro-apoptotic effect by applying Annexin V-PE/7ADD double staining. The results showed that the apoptotic
A

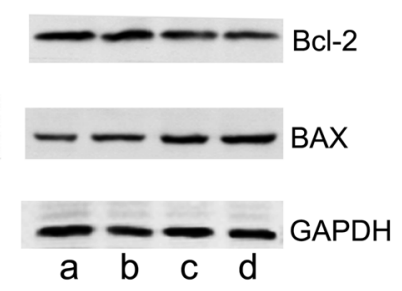

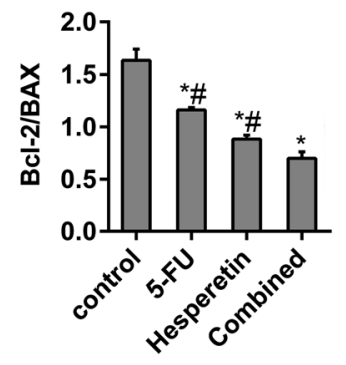

B

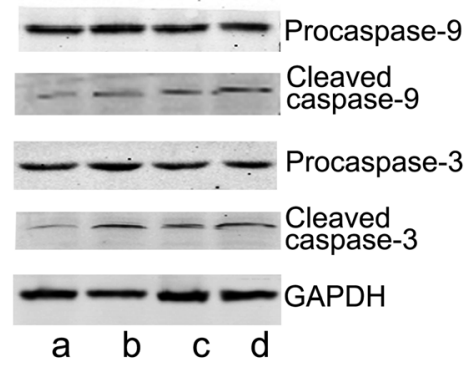

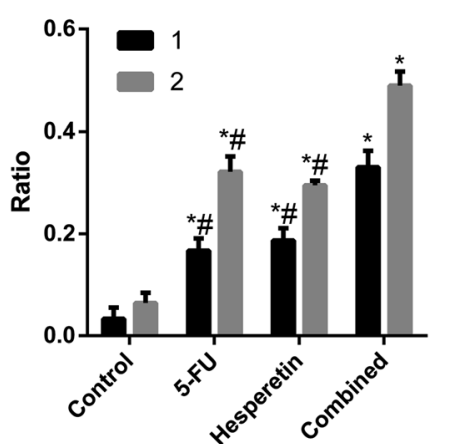

Fig. 5 Hesperetin and 5-FU synergistically affected apoptosis-related protein expression. (A) Bax and Bcl-2 expression levels were detected by western blot. The Bcl-2/Bax ratio was calculated (a, control; b, 5-FU at $20 \mu \mathrm{M}$; c, hesperetin at $200 \mu \mathrm{M} ; \mathrm{d}, 20 \mu \mathrm{M} 5-\mathrm{FU}+200 \mu \mathrm{M}$ hesperetin. a, b, C and $d$ is same in B). (B) Procaspase-9, cleaved caspase-9, procaspase-3, and cleaved caspase-3 levels were assessed (1, cleaved caspase-9/ procaspase-9; 2, cleaved caspase-3/procaspase-3). Data are presented as means \pm SEM $(n=3)$. ${ }^{*} p<0.05$ versus control. ${ }^{\#} p<0.05$ versus combination. 
A
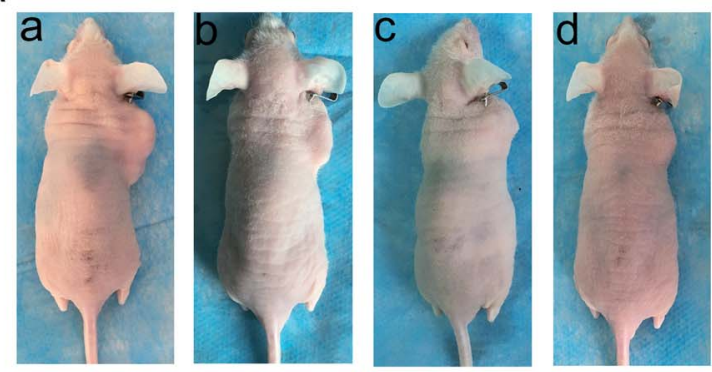

B

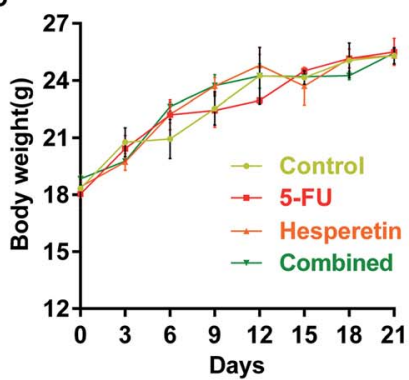

E

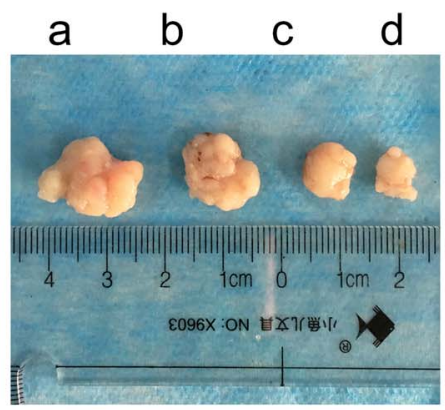

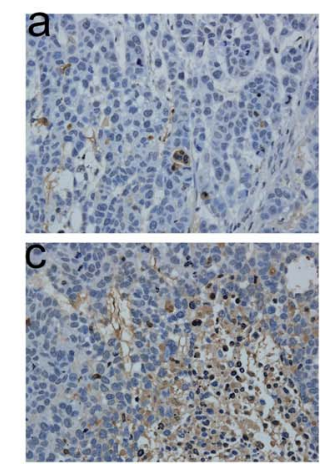

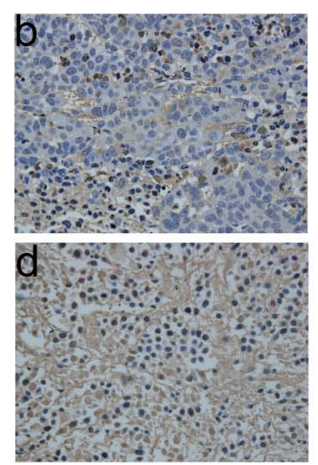

C

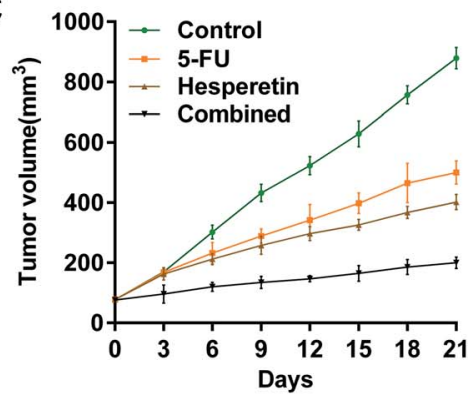

F

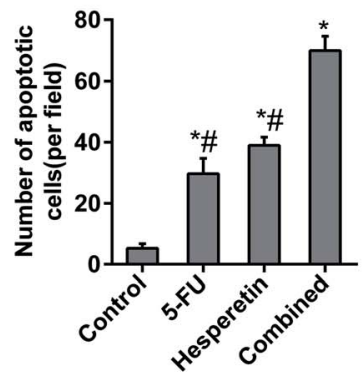

Fig. 6 Hesperetin and 5-FU synergistically inhibited esophageal cancer xenograft growth in vivo. (A) Nude mice were subcutaneously transplanted with Eca-109 cells, then were treated with vehicle (a), 5-FU (b), hesperetin (c), or both (d) (a-d is same in D and E). The weight (B) and tumor size (C) in each group was recorded every 3 days after treatment. (D) The extracted tumor in each group. (E) Apoptotic cells were detected in xenograft tumor tissues using the TUNEL assay $(200 \times)$. The apoptotic cells showed nut-brown nuclei. (F) The apoptotic cells per field in each group were counted on the basis of viewing eight random fields in each glide. All the data are presented as mean \pm SD. * $p<0.05$ versus control. ${ }^{\#} p<0.05$ versus combination.

rate of the combined treatment group $(38.25 \pm 4.52 \%)$ was significantly higher than that of the single drug treatment group ( $200 \mu \mathrm{M}$ hesperetin: $21.38 \pm 3.35 \% ; 20 \mu \mathrm{M}$ 5-FU: $13.91 \pm 2.16 \%)$ $(p<0.05)$ (Fig. 4B). Cell cycle distribution in each group was also analyzed (Fig. 4C). Flow cytometry showed that the combined treatment was associated with drastically increased G0/G1 phase accumulation, indicating that the combined treatment had a stronger effect than either individual treatment did.

To identify the mechanism underlying apoptosis induced by hesperetin combined with 5-FU, we performed western blot analysis to examine the effects of each treatment on the mitochondrial apoptotic pathway. Fig. 5A showed that hesperetin and 5-FU individually or combined increased Bax expression and decreased Bcl-2 expression in the cells, decreasing the Bcl2/Bax ratio. The decrease in the Bcl-2/Bax ratio was greater in the combined treatment group than that in either individual treatment group $(p<0.05)$. The expression levels of cleaved caspase-3 and -9 increased significantly in the individual and combined groups compared with the control group (Fig. 5B). The increase in these levels was also greater in the combined group than that in either individual treatment group $(p<0.05)$.

\section{Hesperetin and 5-FU synergistically suppress tumor development in nude mice}

We next evaluated whether hesperetin combined with 5-FU exerted enhanced anti-tumor effects in vivo. All the mice grew Eca-109 tumor xenografts successfully. No adverse effects were observed in the mice's general appearance, weight, or blood serum alanine aminotransferase, serum aspartate aminotransferase, blood urea nitrogen levels, or serum creatinine levels (Fig. 6A and B, Table 1). As shown in Fig. 6C and D, tumors grew

Table 1 Effect of hesperetin and 5-FU individually or combined on liver and kidney function ${ }^{a}$

\begin{tabular}{lllll}
\hline Group & ALT $\left(\mathrm{U} \mathrm{l}^{-1}\right)$ & AST $\left(\mathrm{U} \mathrm{l}^{-1}\right)$ & Urea $\left(\mu \mathrm{mol} \mathrm{l}^{-1}\right)$ & $\left.\mathrm{Cr}^{-1} \mu \mathrm{mol} \mathrm{l}^{-1}\right)$ \\
\hline Control & $41.33(11.93)$ & $142.67(9.87)$ & $9.21(0.74)$ & $10.67(0.58)$ \\
5-FU & $42.01(4.12)$ & $142.47(10.34)$ & $9.64(0.67)$ & $10.25(0.83)$ \\
Hesperetin & $40.53(2.57)$ & $143.35(7.79)$ & $8.33(1.18)$ & $11.5(0.5)$ \\
Combined & $40.78(2.83)$ & $142.98(2.83)$ & $9.36(0.38)$ & $10.83(1.04)$
\end{tabular}

${ }^{a}$ Data are presented as mean (standard deviation). ALT, alanine aminotransferase; AST, aspartate aminotransferase; BUN, blood urea nitrogen; Cr, serum creatinine. No differences were observed in any laboratory value between the groups $(p>0.05)$. 
Table 2 Inhibitory effect of hesperetin and 5-FU on xenograft volume $^{a}$

\begin{tabular}{lll}
\hline Group & Tumor volume $\left(\mathrm{mm}^{3}\right)$ & Inhibition rate $(\%)$ \\
\hline Control & $813(46.79)$ & \\
5 -FU & $477(80.25)^{* \#}$ & 41.33 \\
Hesperetin & $360(66.36)^{* \#}$ & 55.72 \\
Combined & $157(33.51)^{*}$ & 80.69
\end{tabular}

${ }^{a}$ Data are presented as mean (standard deviation) and inhibition rate. ${ }^{*} p<0.05$ versus control; ${ }^{\#} p<0.05$ versus combination.

Table 3 Inhibitory effect of hesperetin and 5-FU on xenograft weight ${ }^{a}$

\begin{tabular}{lll}
\hline Group & Tumor weight $(\mathrm{g})$ & Inhibition rate $(\%)$ \\
\hline Control & $0.915(0.144)$ & \\
5 -FU & $0.553(0.021)^{* \#}$ & 39.56 \\
Hesperetin & $0.443(0.033)^{* \#}$ & 51.58 \\
Combined & $0.273(0.045)^{*}$ & 70.16 \\
& \\
& \\
&
\end{tabular}

progressively in the control group during treatment period, however, tumor growth was markedly suppressed both in the single and combined treatment groups. Table 2 showed the inhibition rate of tumor volume in the combined treatment group (80.69\%), 5-FU-only group (41.33\%), and hesperetin-only group $(55.72 \%)$ after 21 days of treatment, which clearly indicated that the combined treatment induced greater tumor growth suppression than did hesperetin or 5-FU alone. As shown in Table 3, the mean tumor weight in the combination group was significantly less than that in the hesperetin-only, 5FU-only, or control group. HE staining (not shown) and TUNEL assay of the subcutaneous xenograft sections indicated that hesperetin and 5-FU individually and in combination induced significantly greater apoptosis than the control did (Fig. 6E), and the apoptotic rate in the combination treatment group was greater than that in either individual treatment group (Fig. 6F).

\section{Discussion}

We investigated the effect of hesperetin on esophageal cancer cell proliferation and invasion abilities and assessed relevant molecular mechanisms, and we found that hesperetin not only inhibits Eca-109 cell proliferation and invasion through the PI3K/AKT pathway but also shows synergistic antitumor activity with chemotherapeutic agent 5 -FU in vitro and in vivo in esophageal cancer.

Through flow cytometry with PI staining, we found that hesperetin induced cell cycle arrest at the G0/G1 phase. And EdU assay showed that the number of EdU-positive cells decreased in a concentration-dependent manner. These two assays suggested that hesperetin could inhibit Eca-109 cell growth significantly. Furthermore, western blot analysis revealed that hesperetin functioned as a growth inhibitor of the
PI3K/Akt signaling pathway, a well-characterized cell survival signaling pathway. In numerous cancer tissues and cells, this pathway is overactive, representing an opportunity for drug discovery. ${ }^{23}$ In the present study, we discovered that hesperetin treatment significantly inhibited PI3K and Akt activation and promoted PTEN phosphorylation. The results indicated that hesperetin was able to inhibit proliferation of Eca-109 cells through the PI3K/Akt pathway. Similarly, previous studies have shown that inhibiting the PI3K/Akt signaling pathway by other chemicals can prevent the growth of various cancer cells..$^{24,25}$

The expression of cell cycle regulatory proteins was also detected. We found that hesperetin induced cell cycle arrest by increasing the expression levels of p21 and decreasing cyclin D1. Meanwhile, in addition to its proliferation-inhibiting function, hesperetin played a negative role in the regulation of cell invasion and metastasis. A vital step in cancer metastasis processes is the proteolytic degradation of the extracellular matrix by proteolytic enzymes such as MMPs, and we found that hesperetin significantly decreased the expression levels of MMP-2 and MMP-9. Many studies have reported that MMP-2 and MMP-9 expression were mediated by the PI3K/Akt pathway. ${ }^{26}$ Indeed, Akt activation can lead to cancer invasion and metastasis by stimulating the secretion of MMPs.

The prognosis of esophageal cancer remains poor because most patients present with advanced disease. Chemotherapy is one of the main therapeutic approaches for these patients, and cisplatin-based and/or 5-FU-based combination therapy is routinely used in the clinic. ${ }^{27}$ However, cisplatin- and 5-FUrelated toxicity and resistance remain serious and common issues for many cancer patients. New combination therapies are being explored to maximize therapeutic advantages while reducing side effects and complications. ${ }^{28}$ Among various combination regimens, natural medicines with low toxicity and high efficiency are of particular interest. ${ }^{29}$ Here, we demonstrated that the combination of hesperetin and 5-FU achieved decreasing the dose of 5-FU, which is also effective and safe in the animal models tested.

We studied the synergistic effect of hesperetin and 5-FU on the cell growth and apoptosis of Eca-109 cells in vitro. We found that hesperetin and 5-FU individually exhibited antiproliferative effects on Eca-109 cells and that these effects were significantly enhanced when the cells were treated with these drugs in combination. We also found that hesperetin and 5-FU individually induced significantly greater apoptosis compared with the control and that the combination had a synergistic pro-apoptotic effect. To date, two apoptotic pathways are best understood: the cell death receptor-mediated extrinsic pathway and the mitochondria-mediated intrinsic pathway. Hesperetin has been proven to induce the apoptosis of multiple cancer cells via the intrinsic pathway, including Eca-

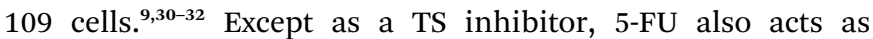
a pyrimidine analogue that incorporates into RNA and DNA, leading to activation of apoptosis involving expression changes of p53 and caspase- $6{ }^{33}$ In this study, we found that the combination of hesperetin and 5-FU could induce Eca-109 cell apoptosis through the intrinsic pathway. The ratio of Bcl-2/Bax dramatically decreased in the combined treatment group 
compared with the individual treatment groups, while an increase was seen for the expression of cleaved caspase-9 and cleaved caspase-3.

Finally, the anti-proliferation effect on Eca-109 xenografts was also enhanced by the combined treatment. The TUNEL assay showed more apoptotic cells in the combined group than in the individual treatment groups or the control group. No obvious adverse effects, such as liver dysfunction and kidney dysfunction, were observed. Thus, hesperetin combined with 5FU exerted a synergistic anti-cancer effect on esophageal cancer in vitro and in vivo.

In conclusion, hesperetin inhibits proliferation and invasion of esophageal cancer cells via the PI3K/Akt pathway, and the combination of hesperetin and 5-FU has synergistic antiapoptotic effects on esophageal cancer cells via the mitochondria-mediated intrinsic pathway without notable toxic effects. Therefore, hesperetin combined with 5-FU may be an effective chemotherapeutic strategy for esophageal cancer.

\section{Conflicts of interest}

The authors have no conflicts of interest.

\section{Acknowledgements}

This work was supported in part by National Natural Science Foundation of China (No. 81572426). This manuscript was edited by the Department of Scientific Publications of the University of Texas MD Anderson Cancer Center, which is supported in part by the National Institutes of Health through Cancer Center Support Grant P30CA016672.

\section{References}

1 L. A. Torre, F. Bray, R. L. Siegel, J. Ferlay, J. Lortet-Tieulent and A. Jemal, Global cancer statistics, Ca-Cancer J. Clin., 2012, 2015(65), 87-108, DOI: 10.3322/caac.21262.

2 R. Siegel, C. DeSantis, K. Virgo, K. Stein, A. Mariotto, T. Smith, D. Cooper, T. Gansler, C. Lerro, S. Fedewa, C. Lin, C. Leach, R. S. Cannady, et al., Cancer treatment and survivorship statistics, Ca-Cancer J. Clin., 2012, 2012(62), 220-241, DOI: 10.3322/caac.21149.

3 B. Keith, Mosby's Oncology Nursing Advisor: A Comprehensive Guide to Clinical Practice, 2016, vol. 58.

4 N. Howlader, A. M. Noone, M. Krapcho, J. Garshell, D. Miller, S. F. Altekruse, C. L. Kosary, M. Yu, J. Ruhl, Z. Tatalovich, A. Mariotto, D. R. Lewis and H. S. Chen, et al., SEER Cancer Statistics Review, 1975-2011, National Cancer Institute, Bethesda, MD, 2013, http://seer.cancer.gov/csr/1975_2011/, based on November, SEER data submission, posted to the SEER web site, April 2014.

5 H. Zeng, R. Zheng, Y. Guo, S. Zhang, X. Zou, N. Wang, L. Zhang, J. Tang, J. Chen, K. Wei, S. Huang, J. Wang, L. Yu, et al., Cancer survival in China, 2003-2005: a population-based study, Int. J. Cancer, 2015, 136, 19211930, DOI: 10.1002/ijc.29227.
6 A. Roohbakhsh, H. Parhiz, F. Soltani, R. Rezaee and M. Iranshahi, Molecular mechanisms behind the biological effects of hesperidin and hesperetin for the prevention of cancer and cardiovascular diseases, Life Sci., 2015, 124, 6474, DOI: 10.1016/j.lfs.2014.12.030.

7 E. J. Choi, Hesperetin induced G1-phase cell cycle arrest in human breast cancer MCF-7 cells: involvement of CDK4 and p21, Nutr. Cancer, 2007, 59, 115-119, DOI: 10.1080/ 01635580701419030.

8 A. A. Alshatwi, E. Ramesh, V. S. Periasamy and P. SubashBabu, The apoptotic effect of hesperetin on human cervical cancer cells is mediated through cell cycle arrest, death receptor, and mitochondrial pathways, Fundam. Clin. Pharmacol., 2013, 27, 581-592, DOI: 10.1111/j.14728206.2012.01061.x.

9 D. Wu, J. Zhang, J. Wang, J. Li, F. Liao and W. Dong, Hesperetin induces apoptosis of esophageal cancer cells via mitochondrial pathway mediated by the increased intracellular reactive oxygen species, Tumour Biol., 2016, 37, 3451-3459, DOI: 10.1007/s13277-015-4176-6.

10 K. Y. Kim, K. I. Park, S. H. Kim, S. N. Yu, S. G. Park, Y. W. Kim, Y. K. Seo, J. Y. Ma and S. C. Ahn, Inhibition of Autophagy Promotes Salinomycin-Induced Apoptosis via Reactive Oxygen Species-Mediated PI3K/AKT/mTOR and ERK/p38 MAPK-Dependent Signaling in Human Prostate Cancer Cells, Int. J. Mol. Sci., 2017, 18, 1088.

11 I. Khan and S. C. Kang, Apoptotic Activity of Lactobacillus plantarum DGK-17-Fermented Soybean Seed Extract in Human Colon Cancer Cells via ROS-JNK Signaling Pathway, J. Food Sci., 2017, 82, 1475-1483.

12 S. Palit, S. Kar, G. Sharma and P. K. Das, Hesperetin Induces Apoptosis in Breast Carcinoma by Triggering Accumulation of ROS and Activation of ASK1/JNK Pathway, J. Cell. Physiol., 2015, 230, 1729-1739, DOI: 10.1002/jcp.24818.

13 L. Cao, X. Chen, X. Xiao, Q. Ma and W. Li, Resveratrol inhibits hyperglycemia-driven ROS-induced invasion and migration of pancreatic cancer cells via suppression of the ERK and p38 MAPK signaling pathways, Int. J. Oncol., 2016, 49, 735-743, DOI: 10.3892/ijo.2016.3559.

14 J. Zhang, X. Wang, V. Vikash, Q. Ye, D. Wu, Y. Liu and W. Dong, ROS and ROS-Mediated Cellular Signaling, Oxid. Med. Cell. Longevity, 2016, 2016, 4350965, DOI: 10.1155/ 2016/4350965.

15 N. Saeed, R. Shridhar, S. Hoffe, K. Almhanna and K. L. Meredith, AKT expression is associated with degree of pathologic response in adenocarcinoma of the esophagus treated with neoadjuvant therapy, J. Gastrointest. Oncol., 2016, 7, 158-165, DOI: 10.3978/j.issn.2078-6891.2015.067.

16 D. A. Altomare and J. R. Testa, Perturbations of the AKT signaling pathway in human cancer, Oncogene, 2005, 24, 7455-7464, DOI: 10.1038/sj.onc.1209085.

17 C. Blanco-Aparicio, O. Renner, J. F. Leal and A. Carnero, PTEN, more than the AKT pathway, Carcinogenesis, 2007, 28, 1379-1386, DOI: 10.1093/carcin/bgm052.

18 M. Shirzad, E. Heidarian, P. Beshkar and M. GholamiArjenaki, Biological Effects of Hesperetin on Interleukin-6/ Phosphorylated Signal Transducer and Activator of 
Transcription 3 Pathway Signaling in Prostate Cancer PC3 Cells, Pharmacogn. Res., 2017, 9, 188-194, DOI: 10.4103/ 0974-8490.204655.

19 S. He, X. Wang, Y. Zhong, L. Tang, Y. Zhang, Y. Ling, Z. Tan, P. Yang and A. Chen, Hesperetin post-treatment prevents rat cardiomyocytes from hypoxia/reoxygenation injury in vitro via activating PI3K/Akt signaling pathway, Biomed. Pharmacother., 2017, 91, 1106-1112, DOI: 10.1016/ j.biopha.2017.05.003.

20 G. D. Kim, Hesperetin Inhibits Vascular Formation by Suppressing of the PI3K/AKT, ERK, and p38 MAPK Signaling Pathways, Prev. Nutr. Food Sci., 2014, 19, 299306, DOI: 10.3746/pnf.2014.19.4.299.

21 D. B. Longley, D. P. Harkin and P. G. Johnston, 5Fluorouracil: mechanisms of action and clinical strategies, Nat. Rev. Cancer, 2003, 3, 330-338, DOI: 10.1038/nrc1074.

22 J. Liu, Z. Wang, K. Wu, J. Li, W. Chen, Y. Shen and S. Guo, Paclitaxel or 5-fluorouracil/esophageal stent combinations as a novel approach for the treatment of esophageal cancer, Biomaterials, 2015, 53, 592-599, DOI: 10.1016/ j.biomaterials.2015.03.009.

23 I. Vivanco and C. L. Sawyers, The phosphatidylinositol 3kinase AKT pathway in human cancer, Nat. Rev. Cancer, 2002, 2, 489-501, DOI: 10.1038/nrc839.

24 Y. Guo, H. Chang, J. Li, X. Y. Xu, L. Shen, Z. B. Yu and W. C. Liu, Thymosin alpha 1 suppresses proliferation and induces apoptosis in breast cancer cells through PTENmediated inhibition of PI3K/Akt/mTOR signaling pathway, Apoptosis, 2015, 20, 1109-1121, DOI: 10.1007/s10495-0151138-9.

25 M. Block, S. Fister, G. Emons, S. Seeber, C. Grundker and A. R. Gunthert, Antiproliferative effects of antiestrogens and inhibitors of growth factor receptor signaling on endometrial cancer cells, Anticancer Res., 2010, 30, 20252031.
26 W. G. Jiang, A. J. Sanders, M. Katoh, H. Ungefroren, F. Gieseler, M. Prince, S. K. Thompson, M. Zollo, D. Spano, P. Dhawan, D. Sliva, P. R. Subbarayan, M. Sarkar, et al., Tissue invasion and metastasis: Molecular, biological and clinical perspectives, Semin. Cancer Biol., 2015, 35(suppl.), S244-S275, DOI: 10.1016/j.semcancer.2015.03.008.

$27 \mathrm{H}$. Kato and M. Nakajima, Treatments for esophageal cancer: a review, Gen. Thorac. Cardiovasc. Surg., 2013, 61, 330-335, DOI: 10.1007/s11748-013-0246-0.

28 D. Lin and L. Leichman, The current status of neoadjuvant therapy for esophageal cancer, Semin. Thorac. Cardiovasc. Surg., 2014, 26, 102-109, DOI: 10.1053/ j.semtcvs.2014.07.002.

$29 \mathrm{~J}$. Uzoigwe and E. R. Sauter, Cancer prevention and treatment using combination therapy with plant- and animal-derived compounds, Expert Rev. Clin. Pharmacol., 2012, 5, 701-709, DOI: 10.1586/ecp.12.62.

30 J. Zhang, J. Song, D. Wu, J. Wang and W. Dong, Hesperetin induces the apoptosis of hepatocellular carcinoma cells via mitochondrial pathway mediated by the increased intracellular reactive oxygen species, ATP and calcium, Med. Oncol., 2015, 32, 101, DOI: 10.1007/s12032-015-0516-z.

31 J. Zhang, D. Wu, V. Vikash, J. Song, J. Wang, J. Yi and W. Dong, Hesperetin Induces the Apoptosis of Gastric Cancer Cells via Activating Mitochondrial Pathway by Increasing Reactive Oxygen Species, Dig. Dis. Sci., 2015, 60, 2985-2995, DOI: 10.1007/s10620-015-3696-7.

32 A. Adan and Y. Baran, Fisetin and hesperetin induced apoptosis and cell cycle arrest in chronic myeloid leukemia cells accompanied by modulation of cellular signaling, Tumour Biol., 2016, 37, 5781-5795, DOI: 10.1007/s13277015-4118-3.

33 N. Zhang, Y. Yin, S. J. Xu and W. S. Chen, 5-Fluorouracil: mechanisms of resistance and reversal strategies, Molecules, 2008, 13, 1551-1569. 